\title{
Feasibility of Wireless Power Transfer for Electrification of Transportation: Techno-economics and Life Cycle Assessment
}

\author{
Jason C. Quinn, Braden J. Limb, Zeljko Pantic, Paul Barr, Regan Zane \\ Utah State University \\ Logan, UT, USA \\ jason.quinn@usu.edu \\ Thomas H. Bradley \\ Colorado State University \\ Fort Collins, CO, USA
}

\begin{abstract}
Integration of wireless power transfer (WPT) systems in roadways and vehicles represents a promising alternative to traditional internal combustion transportation systems. The economic feasibility and environmental impact of WPT applied to the transportation system is evaluated through the development of engineering system models. For a $20 \%$ penetration of the WPT technology in vehicles, results show a $20 \%$ reduction in air pollutants, $10 \%$ reduction in energy use and $\mathrm{CO}_{2}$ emissions and a societal level payback (defined as total cost of ownership savings compared to a traditional vehicle equal to roadway infrastructure) of 3 years. The modeled system covers $86 \%$ of all traffic in the US, impacts $40 \%$ of all roadways and shifts $\mathbf{\$ 1 8 0}$ billion per year from oil production to jobs in local power generation and development, construction, and maintenance of electrified roadways and new electric vehicles. Results on model sensitivity to energy prices, payback as a function of penetration, and trucking vs light duty use are presented.
\end{abstract}

Keywords- Electric vehicles, Wireless Power Transfer, Sustainability, economics

\section{INTRODUCTION}

The need for alternative vehicle technologies has become more prevalent with increased pressure to decrease dependence on foreign energy. Each year transportation is a primary consumer of fossil fuel in the United States accounting for 27 quadrillion Btu's of energy. This equates to $27.7 \%$ of the total energy and $78 \%$ of the petroleum used in the United States annually [1]. A variety of alternative electrified transportation technologies are being investigated including electric vehicles (EVs), plug-in hybrid electric vehicles (PHEVs), and hybrid electric vehicles (HEVs). Consumer acceptability and market penetration of these vehicles has been limited due to the restricted range and total ownership costs as compared to traditional internal combustion (ICE) vehicles. Preliminary solutions to consumer concerns have centered on the energy storage onboard, specifically the battery. Challenges associated with satisfying range requirements through increased battery size include an increased cost and weight of the vehicle [2]. In an effort to move away from the dependence on batteries there has been a growing interest towards the implementation of in-motion wireless power transfer (WPT) with EVs. The economic feasibility and environmental impact of this solution has not been fully explored.

System models have previously been used to understand the potential impact of alternative transportation vehicles including PHEVs, EVs, and HEVs with a variety of architectures (fuel cell, series or parallel hybrid). Previous modeling efforts have focused on vehicle performance, prediction, safety, structural integrity, component testing and validation, architecture optimization, techno-economics, environmental impact and forecasting of consumer acceptability and market penetration rates [3-8]. Systems models have been developed and applied to WPT research and development for the optimization of charging systems used in various application ranging from visual prosthesis [9] to robots [10]. Limited modeling and assessment work has been done bridging WPT and transportation. Understanding the potential impact of WPT applied to the transportation infrastructure requires the development of dynamic systems models that can be leveraged for techno-economic analyses, life-cycle assessments, systems optimization, and prediction of market penetration rates.

The integration of WPT into transportation systems has the potential to dramatically impact vehicle architectures and transportation systems. This work develops system models that are leveraged to understand the techno-economic feasibility and environmental impact of in-motion WPT applied to vehicle transportation in the US. Foundational 
modeling includes all components required for the deployment of a WPT system and includes a direct comparison to traditional internal combustion vehicles. The modeled system covers $86 \%$ of all traffic in the US, impacts $40 \%$ of all roadways, and includes a sensitivity to fuel and energy costs. Results highlight the potential impact and societal payback of WPT applied to transportation as compared to current traditional systems. Discussion focuses on the economic impact, impact of penetration, and potential demonstration platforms.

\section{OVERVIEW OF MODELING METHODS}

The technology being evaluated is based on the development of roadway infrastructure and vehicle components that support in-motion WPT. A conceptual schematic of the technology is illustrated in Fig. 1. To evaluate the technology, vehicle models were developed and leveraged to understand the economic and environmental impact of the technology.

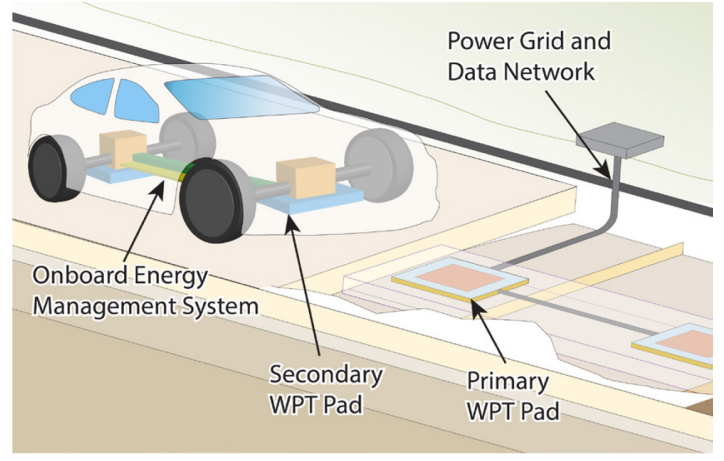

Fig. 1. System concept for integration of WPT into an electrified transportation system

The evaluation of the technology includes a detailed assessment of the cost and environmental impact for the deployment of WPT for EV operation in the US and is directly compared to traditional internal combustion vehicles. The analysis includes a detailed return on investment at the societal level for development of infrastructure to support deployment of wireless power transfer. Detailed assumptions for the vehicle and roadway systems are presented in the following sections. Advanced vehicle models were developed and leveraged with standard drive cycles to better understand energy consumption. Results from this work are directly compared to initial assumptions and highlight the need for higher fidelity modeling.

\section{A. Vehicle Modeling}

Two different vehicle architecture models were developed, 1) traditional ICE vehicle and 2) an all-electric WPT vehicle. In each of these architectures two different vehicles where modeled, light duty and truck, to accurately capture the majority of the vehicles on US roadways. The vehicle model for a the light duty vehicles focused on the energy requirements of the vehicles operated at $60 \mathrm{mph}$ with a frontal area of $1.35 \mathrm{~m}^{3}$, assumes a drive train efficiency of $85 \%$ and assumes the vehicle is operated on a zero percent grade road.

ICE vehicle: The primary assumptions for the ICE vehicle included a vehicle weight of $2,000 \mathrm{~kg}$, an engine efficiency of $28 \%$, purchase price of $\$ 31,252$, maintenance cost of $8 \%$ of the purchase price over the life of the vehicle and an energy requirement at the wheel of $425 \mathrm{Wh} \mathrm{mi}^{-1}$.

WPT vehicle: The primary assumptions for the WPT vehicle included a vehicle weight of $1,400 \mathrm{~kg}$, an electric energy delivery efficiency of $84 \%$ (includes WPT and energy storage), purchase price of $\$ 21,877$, maintenance cost of $4 \%$ of the purchase price over the life of the vehicle and an energy requirement at the wheel of $383 \mathrm{Wh} \mathrm{mi}^{-1}$.

Truck modeling: The truck was modeled similarly for the two vehicle power systems as the weight of the vehicle is dominated by the load of the vehicle and the assumed cost of the two systems are expected to be similar. The purchase price of the truck is assumed to be $\$ 250,000$, the required energy at the wheels is $870 \mathrm{Wh} \mathrm{mi}^{-1}$, and the vehicle efficiencies and maintenance costs are assumed to be the same as above.

\section{B. Roadway Infrastructure}

A total of 2.6 million miles of paved roadways exist in the US and are classified into three different classifications: interstate, urban and rural. The breakdown of paved roads are $1.7 \%, 38.4 \%$, and $59.8 \%$ for interstate, urban and rural, respectively. Even though rural roads represent the majority of the number of miles that are paved in the US, only $14 \%$ of the miles (320 million miles) driven in the US are performed on this classification of roadway with $86 \%$ performed on urban (1.4 trillion miles) and interstate (614 million miles) roadways [11]. Based on feasibility of rollout, the modeling work excludes the upgrading of rural roads and only includes interstate and urban roadways. The assumed upgrade costs for integrating WPT into the roadway is $\$ 2.4$ million lane $\mathrm{e}^{-1} \mathrm{mile}^{-1}$. WPT pads of $25 \mathrm{~kW}$ are assumed resulting in a requirement of $33 \%$ of the total interstate roadway covered. It is assumed that 2 lanes, one in each direction, are converted. The assumed costs include all WPT electronics and electric power delivery infrastructure. Urban roads are assumed to cost the same as interstate roads for upgrading. The covered length is decreased to $6 \%$ based on strategic placement of charging systems [12]. The decrease in total roadway coverage assumes vehicles can charge at stationary locations when not in use. Roadway costs represent a dynamic variable with some roadways such as intercity interstate systems costing significantly more that the assumed and other roadways costs less than what is assumed. The $\$ 2.4$ million lane mile $^{-1}$ is expected to be a realistic cost for retrofit of existing roadways and not the expansion of existing roadways.

\section{Life Cycle Assumptions}

The environmental impact of the two systems, traditional ICE and WPT, is evaluated. Life cycle inventory data from the ANL GREET model is integrated with system modeling 
results for fuel consumption and electrical consumption [13]. The life cycle emissions is limited to the emissions associated with the use of the vehicle and excludes the emissions associated with manufacturing of the vehicles and the upgrading of the roadway systems. An ICE vehicle and ICE

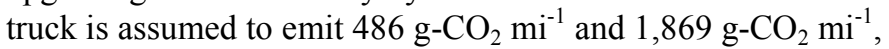
respectively. The emissions from electrical production are assumed to be $545 \mathrm{~g}-\mathrm{CO}_{2} \mathrm{kWhr}^{-1}$ corresponding to a mix of technologies predominantly composed of coal (39\%), natural gas (27\%), and nuclear (18\%). Criteria pollutants, VOC, CO, NOx, PM10, PM2.5, and SOx are also included in the assessment.

\section{Techno-economic Assumptions}

The techno-economic assessment is performed on a societal level with a payback period calculated as a function of penetration rate. The payback period is defined as the time it takes for the cost of the roadway infrastructure to be repaid through cost saving associated with the operation, maintenance and purchase saving associated with the WPT vehicle architecture and roadway. The assessment includes sensitivity to fuel costs. The baseline fuel price is set at $\$ 4.07$ $\mathrm{gal}^{-1}$ with the high and low price set to $\$ 5.89 \mathrm{gal}^{-1}$ and $\$ 2.30$ $\mathrm{gal}^{-1}$. The baseline electric cost is set at $\$ 0.107 \mathrm{kWhr}^{-1}$. The defined payback period represents an initial understanding of the feasibility of the technology

\section{RESULTS AND DISCUSSION}

The results and discussion are divided into three sections, 1) techno-economic results, 2) environmental impact, and 3) dynamic vehicle modeling energy consumption.

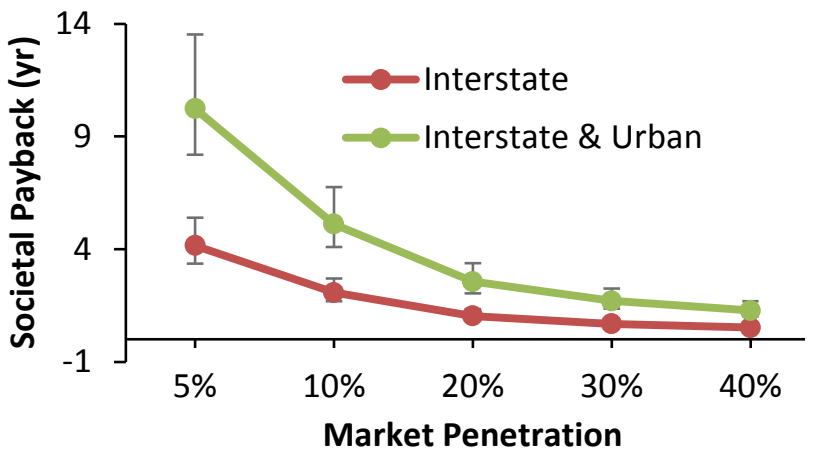

Fig. 2. Societal payback as a function of market penetration. Two electrification scenarios are presented, 1) interstate roadway system (red) and 2) interstate and urban roadways (green). Error bars represent sensitivity to fuel prices

The techno-economic results for the societal payback are presented in Fig. 2 as a function of market penetration rate. Results for the electrification of two roadway systems are presented, 1) interstate and 2) interstate and urban. The error bars illustrate the impact of fuel price on the return on investment. The analysis for the interstate system represents the smallest initial capital investment, $\$ 37$ billion, and covers $28 \%$ of the miles driven in the US. The second modeled scenario requires an initial capital investment of $\$ 230$ billion and covers $86 \%$ of the miles driven in the US.
The societal payback at a $20 \%$ penetration for baseline case which includes electrification of the interstate and urban roadways is a promising 2.6 years. The payback for electrification of only the interstate system at a $20 \%$ penetration is 1.04 years. The promising payback periods are driven by the total number of miles driven in the US, 2.6 trillion annually, combined with the decreased operating costs of the WPT vehicle. The decreased operating costs are due to the high efficiency of energy transfer and the low cost of electrical energy compared to petroleum based fuel. Fuel price is shown to dramatically impact the payback at small penetration rates with the impact decreasing as penetration rate increases. The results of the analysis are driven by the low cost of delivering energy to the wheels of an EV compared to a traditional ICE vehicle. This is primarily driven by the high efficiency of electrical production, delivery, and use compared to that of an ICE based architecture. The economic results illustrate the financial incentive to pursue electrification of transportation. It is acknowledged that rollout of an electrical system represents a major challenge; however the economics of the WPT system are promising. It is expected that small scale systems such as city transportation systems or closed campus transportation would be ideal for demonstration facilities.

The environmental impact of WPT integrated with EVs is directly compared to traditional ICE transportation based on a system boundary that includes all emissions associated with energy production and use in the two vehicle architectures, ICE and WPT EV. The results are separated into two metrics, global warming potential $\left(\mathrm{g}-\mathrm{CO}_{2} \mathrm{mi}^{-1}\right)$ and criteria pollutants (VOC, CO, NOx, PM10, PM2.5, and SOx). Results for the light duty vehicle are presented in Fig. 3.

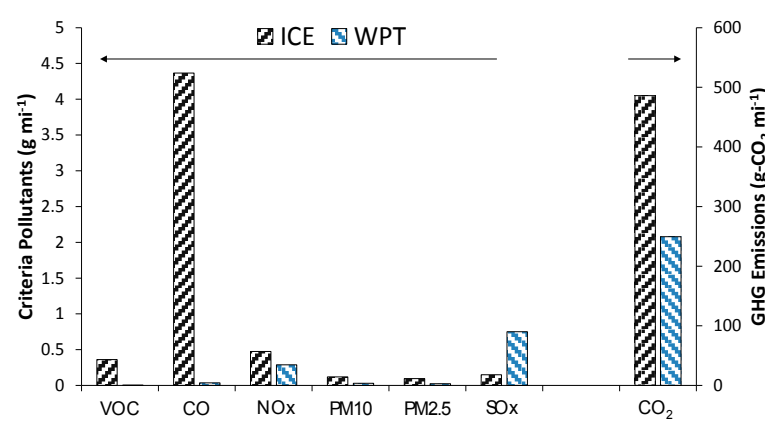

Fig. 3. Environmental impact of a light duty ICE vehicle compared to a WPT EV.

The WPT EV has a $49 \% \mathrm{CO}_{2}$ savings compared to a traditional ICE vehicle. Further, the emissions associated with the WPT EV are centralized and not emitted at the point of consumption. For areas that have poor air quality due to transportation congestion, the WPT EV represents a technology that not only reduces the majority of emissions but relocates them to the point of electrical production. In terms of criteria pollutants, SOx is the only one that increases. This is primarily driven by the amount of SOx produced in coal based 
power which represents $39 \%$ of the power for the WPT EV. Significant reductions are seen in the other criteria pollutants, specifically a $99 \%$ reduction in VOC and CO, a $75 \%$ reduction in PM10 and PM2.5, and a 40\% reduction in NOx.

Combining these results with the total number of miles driven in the US by light duty vehicles and results from the analysis associated with WPT EV trucks, the total amount of $\mathrm{CO}_{2}$ emissions decreases by $10.1 \%$ (134 million tons per year savings) assuming a $20 \%$ penetration.

The analysis performed is limited to a fixed vehicle energy consumption primarily a function of an average vehicle speed and frontal area. The initial modeling efforts were focused on understanding the potential impact of the technology and highlighting areas for research and development. The promising economic and environmental impact results highlight the need to improve the modeling work being performed. Dynamic vehicle models were put together and used to understand more accurately the energy consumption based on dynamic drive cycles. Vehicle architectures included $\mathrm{BEV}, \mathrm{HEV}$, and ICE. Energy consumption of these vehicles was evaluated based on 12 different drive cycles. A vehicle energy consumption result from the simulation of 53 different combinations of vehicle architectures and drive cycles compared to the assumed vehicle energy consumption in this study is presented in Fig. 4.

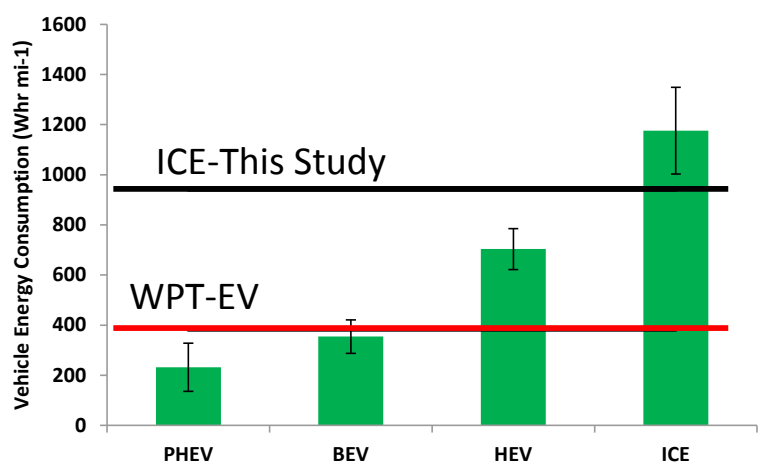

Fig. 4. Comparison of vehicle energy assumed in this study to results from dynamic vehicle modeling with various drive cycles

The vehicle energy consumption assumed in this study based on static speed vehicle modeling is within the range of vehicle energy consumption based on dynamic drive cycles for the WPT-EV. The assumed ICE vehicle energy consumption is slightly lower than the results from dynamic vehicle modeling.

\section{CONCLUSION}

The integration of WPT with EVs represents a promising alternative to traditional ICE vehicles. Modeling work that integrates dynamic vehicle models with technology advancements enables a high level assessment of the potential impact as compared to traditional and alternative technologies. The results from this work show on a societal level, infrastructure payback can be achieved with minimal penetration. The environmental results show the technology to be beneficial in terms of global warming impact and all criteria pollutants with the exception of SOx. WPT addresses some of the limitations that are currently plaguing EVs, specifically range anxiety. The analysis presented shows WPT applied to transportation represents a promising alternative in terms of economics and environmental impact. Integrating dynamic vehicle modeling with consumer driving patterns will facilitate further evaluation and optimization of integrating WPT with transportation systems. Data feedback from modeling can further be leveraged to focus research and development of system components driving towards commercially viable solutions on small and national transportation scale systems.

\section{ACKNOWLEDGMENT}

The authors acknowledge support from Danna Quinn.

\section{REFERENCES}

[1] U. S. Department of Energy, "How much oil consumed in the United States comes from foreign sources?," Energy Efficiency and Renewable Energy, Ed., ed, 2014.

[2] A. Gil and J. Taiber, "A Literature Review in Dynamic Wireless Power Transfer for Electric Vehicles: Technology and Infrastructure Integration Challenges," in Sustainable Automotive Technologies 2013, ed: Springer, 2014, pp. 289-298.

[3] B. M. Al-Alawi and T. H. Bradley, "Review of hybrid, plug-in hybrid, and electric vehicle market modeling Studies," Renewable and Sustainable Energy Reviews, vol. 21, pp. 190-203, 2013. P. Balducci, "Plug-in hybrid electric vehicle market penetration scenarios," PNNL-17441 Report. Pacific Northwest National Laboratory. Richland, WA, 2008. M. A. Delucchi and T. E. Lipman, "An analysis of the retail and lifecycle cost of battery-powered electric vehicles," Transportation Research Part D: Transport and Environment, vol. 6, pp. 371-404, 2001.

D. W. Gao, C. Mi, and A. Emadi, "Modeling and simulation of electric and hybrid vehicles," Proceedings of the IEEE, vol. 95, pp. 729-745, 2007.

B. M. Geller, "Increased Understanding of Hybrid Vehicle Design through Modeling, Simulation, and Optimization," Colorado State University, 2010.

D. Karbowski, A. Rousseau, S. Pagerit, and P. Sharer, "Plug-in vehicle control strategy: from global optimization to real time application," in 22nd Electric Vehicle Symposium, EVS22, Yokohama, Japan, 2006.

X. Li, Y. Yang, and Y. Gao, "Visual prosthesis wireless energy transfer system optimal modeling," Biomedical engineering online, vol. 13, p. 3, 2014.

A. Kurs, A. Karalis, R. Moffatt, J. D. Joannopoulos, P. Fisher, and M. Soljačić, "Wireless power transfer via strongly coupled magnetic resonances," Science, vol. 317, pp. 83-86, 2007.

[11] US Department of Energy, Transportation energy data book, 2014.

[12] Z. Pantic, S. Bai, and S. M. Lukic, "Inductively coupled power transfer for continuously powered 
electric vehicles," in Vehicle Power and Propulsion Conference, 2009. VPPC'09. IEEE, 2009, pp. 12711278.

[13] M. Wang, and Elgowainy, A., "Operating Manual for GREET: Version 1.7," Center for Transportation
Research, Energy Systems Division, Argonne National Laboratory2005. 